\title{
Bedaquiline as a potential agent in the treatment of $M$. intracellulare and M. avium infections
}

To the Editor:

Recently in this journal, SAlfinger and Migliori [1] discussed the accessibility of bedaquiline drug susceptibility testing and the analysis of minimum inhibitory concentrations (MICs) for strains of Mycobacterium tuberculosis. As the recommended treatments for diseases caused by M. avium, and especially $M$. intracellulare, have high failure rates $[2,3]$, we measured the MIC of bedaquiline in 20 clinical isolates of these species.

Bedaquiline is a new anti-tuberculous drug, recently licensed for the treatment of multidrug-resistant M. tuberculosis infections. It belongs to the diarylquinoline group and acts by inhibiting mycobacterial F1Fo-ATP synthase [4]. Bedaquiline has strong in vitro activity against $M$. tuberculosis, including resistant strains $[5,6]$, and an increasing body of post-licensing evidence suggests it has excellent efficacy in the treatment of multidrug-resistant and extensively drug-resistant TB [7-10]. Bedaquiline is characterised pharmacologically by its excellent intracellular bactericidal activity and high accumulation rate [4]. For conventional drugs, in vitro drug susceptibility testing of M. avium-intracellulare complex (MAC) strains is currently recommended only after treatment failure, and the methods are not yet well established $[1,2,5,11]$.

For decades, our laboratory has routinely determined the MICs of first- and second-line TB drugs in multidrug-resistant TB and nontuberculous mycobacteria (NTM) including MAC strains [12]. Here, we present the in vitro susceptibility testing results of 11 clinical strains of $M$. intracellulare and nine of M. avium, isolated from patients with pulmonary NTM disease in our centre between 2008 and 2015. MICs were determined using a modified agar dilution method on Middlebrook 7H10 agar, as described elsewhere [13]. MIC was defined as the lowest drug concentration that inhibited at least $99 \%$ of the bacterial proportion after a two-fold serial dilution of the respective drug.

Results are shown in table 1 . Bedaquiline had a low MIC in nine M. intracellulare strains $\left(0.06 \mu \mathrm{g} \cdot \mathrm{mL}^{-1}\right)$. For M. avium, the MIC was $0.12 \mu \mathrm{g} \cdot \mathrm{mL}^{-1}$ in five strains and $0.06 \mu \mathrm{g} \cdot \mathrm{mL}^{-1}$ in four. There was a nonsignificant trend towards a lower MIC in $M$. intracellulare than in M. avium (Chi-squared test: $\mathrm{p}=0.066$ ). These MIC values were only slightly higher than those published for strains of $M$. tuberculosis [14]. In 2007, Huitric et al. [14] tested 17 M. avium strains and five M. intracellulare strains and also found low MIC values.

Bedaquiline could be an effective candidate in the second-line treatment of mycobacterial disease caused by MAC. Philley et al. [15] reported its successful use in the salvage therapy of six patients with MAC infection of the lungs without differentiated species information. In general, when treatment of NTM infections is indicated, the duration of treatment is $>12$ months $[2,11]$. It is reasonable to consider the effects of long-term treatment with new drugs used in relapsing or complicated NTM disease. Regarding toxicity, Lewis et al. [7] recently published a first experience of long-term bedaquiline use (18 months) in a case of extensively drug-resistant $\mathrm{TB}$, reporting that no clinical side effects had occurred during the whole observation period.

In view of the in vitro data, bedaquiline seems to be a good candidate for severe or relapsing diseases caused by $M$. intracellulare and $M$. avium, respectively. Clinical trials are warranted to confirm this potential use.

@ERSpublications

Bedaquiline is a potential agent to treat severe or relapsing $M$. avium-intracellulare complex infections http://ow.ly/n1eR308pERh

Cite this article as: Vesenbeckh S, Schönfeld N, Krieger D, et al. Bedaquiline as a potential agent in the treatment of $M$. intracellulare and M. avium infections. Eur Respir J 2017; 49: 1601969 [https://doi.org/ 10.1183/13993003.01969-2016]. 
TABLE 1 Minimum inhibitory concentrations (MICs) of bedaquiline for 20 strains of M. avium-intracellulare complex $(p=0.066)$

\begin{tabular}{llccc} 
& $\mathrm{n}(\%)$ & \multicolumn{3}{c}{$\mathrm{MIC} \boldsymbol{\mu g} \cdot \mathrm{mL}^{-1}$} \\
\cline { 3 - 4 } & & $\mathbf{0 . 0 6}$ & $\mathbf{0 . 1 2}$ & $\mathbf{0 . 2 5}$ \\
\hline M. avium & $9(45)$ & $4 / 9(44)$ & $5 / 9(56)$ & - \\
M. intracellulare & $11(55)$ & $9 / 11(82)$ & $1 / 11(9)$ & $1 / 11(9)$
\end{tabular}

Silvan Vesenbeckh ${ }^{1}$, Nicolas Schönfeld ${ }^{1}$, David Krieger ${ }^{1}$, Gudrun Bettermann ${ }^{2}$, Torsten Thomas Bauer ${ }^{1,3}$, Holger Rüssmann ${ }^{2}$ and Harald Mauch ${ }^{2}$

${ }^{1}$ Klinik für Pneumologie, Lungenklinik Heckeshorn, HELIOS Klinikum Emil von Behring, Berlin, Germany. ${ }^{2}$ Institut für Mikrobiologie, Immunologie und Laboratoriumsmedizin, HELIOS Klinikum Emil von Behring, Berlin, Germany. ${ }^{3}$ Deutsches Zentralkomitee zur Bekämpfung der Tuberkulose (DZK), Berlin, Germany.

Correspondence: Silvan Vesenbeckh, Klinik für Pneumologie, Lungenklinik Heckeshorn, HELIOS Klinikum Emil von Behring, Walterhoeferstr. 11, 14165 Berlin, Germany. E-mail: silvan.vesenbeckh@helios-kliniken.de

Received: Oct 062016 | Accepted after revision: Dec 042016

Conflict of interest: None declared.

\section{References}

1 Salfinger M, Migliori GB. Bedaquiline: 10 years later, the drug susceptibility testing protocol is still pending. Eur Respir J 2015; 45: 317-321.

2 Schönfeld N, Haas W, Richter E, et al. Empfehlungen zur Diagnostik und Therapie nichttuberkuloser Mykobakteriosen des Deutschen Zentralkomitees zur Bekampfung der Tuberkulose (DZK) und der Deutschen Gesellschaft fur Pneumologie und Beatmungsmedizin (DGP) [Recommendations for diagnosis and treatment of nontuberculous mycobacterioses of the German Central Committee against tuberculosis and the German Respiratory Society]. Pneumologie 2013; 67: 605-633.

$3 \mathrm{Xu} \mathrm{HB}$, Jiang RH, Li L. Treatment outcomes for Mycobacterium avium complex: a systematic review and meta-analysis. Eur J Clin Microbiol Infect Dis 2014; 33: 347-358.

4 Hards K, Robson JR, Berney M, et al. Bactericidal mode of action of bedaquiline. J Antimicrob Chemother 2015; 70: 2028-2037.

5 Keller PM, Homke R, Ritter C, et al. Determination of MIC distribution and epidemiological cutoff values for bedaquiline and delamanid in Mycobacterium tuberculosis using the MGIT 960 system equipped with TB eXiST. Antimicrob Agents Chemother 2015; 59: 4352-4355.

6 Diacon AH, Pym A, Grobusch MP, et al. Multidrug-resistant tuberculosis and culture conversion with bedaquiline. N Engl J Med 2014; 371: 723-732.

7 Lewis JM, Hine P, Walker J, et al. First experience of effectiveness and safety of bedaquiline for 18 months within an optimised regimen for XDR-TB. Eur Respir J 2016; 47: 1581-1584.

8 Pontali E, Sotgiu G, D'Ambrosio L, et al. Bedaquiline and multidrug-resistant tuberculosis: a systematic and critical analysis of the evidence. Eur Respir J 2016; 47: 394-402.

9 Tadolini M, Garcia-Prats AJ, D’Ambrosio L, et al. Compassionate use of new drugs in children and adolescents with multidrug-resistant and extensively drug-resistant tuberculosis: early experiences and challenges. Eur Respir J 2016; 48: 938-943.

10 Tadolini M, Lingtsang RD, Tiberi S, et al. First case of extensively drug-resistant tuberculosis treated with both delamanid and bedaquiline. Eur Respir J 2016; 48: 935-938.

11 Griffith DE, Aksamit T, Brown-Elliott BA, et al. An official ATS/IDSA statement: diagnosis, treatment, and prevention of nontuberculous mycobacterial diseases. Am J Respir Crit Care Med 2007; 175: 367-416.

12 Radenbach KL. Diagnostische und therapeutische Fortschritte bei nichttuberkulosen Mykobakteriosen [Diagnostic and therapeutic progress in nontuberculous mycobacterioses]. Prax Klin Pneumol 1985; 39: 43-49.

13 Schönfeld N, Bergmann T, Vesenbeckh S, et al. Minimal inhibitory concentrations of first-line drugs of multidrug-resistant tuberculosis isolates. Lung India 2012; 29: 309-312.

14 Huitric E, Verhasselt $\mathrm{P}$, Andries $\mathrm{K}$, et al. In vitro antimycobacterial spectrum of a diarylquinoline ATP synthase inhibitor. Antimicrob Agents Chemother 2007; 51: 4202-4204.

15 Philley JV, Wallace RJ Jr, Benwill JL, et al. Preliminary results of bedaquiline as salvage therapy for patients with nontuberculous mycobacterial lung disease. Chest 2015; 148: 499-506. 